\title{
Subtotal Esophagectomy
}

National Cancer Institute

\section{Source}

National Cancer Institute. Subtotal Esophagectomy. NCI Thesaurus. Code C51579.

Surgical removal of part of the esophagus. 